\title{
Temperature dependence of the upper critical field of an anisotropic singlet superconductivity in a square lattice tight-binding model in parallel magnetic fields
}

\author{
Hiroshi Shimahara and Kentaro Moriwake \\ Department of Quantum Matter Science, ADSM, Hiroshima University, Higashi-Hiroshima 739-8530, Japan
}

(January 26, 2002)

\begin{abstract}
Upper critical field parallel to the conducting layer is studied in anisotropic type-II superconductors on square lattices. We assume enough separation of the adjacent layers, for which the orbital pairbreaking effect is suppressed for exactly aligned parallel magnetic field. In particular, we examine the temperature dependence of the critical field $H_{c}(T)$ of the superconductivity including the FuldeFerrell-Larkin-Ovchinnikov (FFLO or LOFF) state, in which the Cooper pairs have non-zero centerof-mass momentum q. In the system with the cylindrically symmetric Fermi-surface, it is known that $H_{\mathrm{c}}(T)$ of the $d$-wave FFLO state exhibits a kink at a low temperature due to a change of the direction of $\mathbf{q}$ in contrast to observations in organic superconductors. It is shown that the kink disappears when the Fermi-surface is anisotropic to some extent, since the direction of $\mathbf{q}$ is locked in an optimum direction independent of the temperature.
\end{abstract}

Recently, upturn of the curve of the upper critical field as a function of the temperature has been observed in organic superconductors when the magnetic field is applied in the directions parallel to the conducting layers [1] 6]. This behavior has often been discussed [7] in connection with a possibility of the Fulde-Ferrell-LarkinOvchinnikov (FFLO) state [14,15]. It is known by many theoretical calculations [16,17, 9 , 9 that the parallel critical field exhibits upturn at low temperatures for the FFLO state in some models. However, in $d$-wave pairing, the curve of the critical field has a kink above the temperatures at which the upturn occurs when the orbital pair-breaking effect is negligible [18, 11, 19, 13, althought the kink has not been observed yet. In this paper, we show that the kink is suppressed by anisotropy of the Fermi-surfaces of some extent.

Firstly, we briefly review theories and experiments which are concerned with the present theory. The superconductivity in type II superconductor is suppressed by applied magnetic fields due to both the Lorentz force and Zeeman energy for the singlet pairing. However, the Lorentz force is not effective, when the magnetic field is applied in directions parallel to the conducting layers, since the spatial motion of electron is restricted in the conducting layers. In this situation, the pair-breaking is mainly due to the Zeeman energy, and the upper critical field is known as the Chandrasekhar and Clogston limit (Pauli paramagnetic limit) 20,21.

For example, for $s$-wave pairing, the critical field at zero temperature $H_{\mathrm{P}}^{(0)}$ is estimated from the zero field transition temperature $T_{\mathrm{c}}^{(0)}$ by a formula $\mu_{0} H_{\mathrm{P}}^{(0)} \approx 1.25 \times$ $k_{\mathrm{B}} T_{\mathrm{c}}^{(0)}$ in the weak coupling limit, where $\mu_{0}$ is the electron magnetic moment. The critical field is maximum at $T=0$ with $\left[\mathrm{d} H_{\mathrm{P}}(T) / \mathrm{d} T\right]_{T=0}=0$, and it is upwards convex at low temperature $\left(\mathrm{d}^{2} H_{\mathrm{P}}(T) / \mathrm{d} T^{2}<0\right)$.

Fulde and Ferrell [14], and independently Larkin and Ovchinnikov 15] predicted that the critical field can ex- ceed the original Pauli paramagnetic limit by a pairing state with a non-zero center-of-mass momentum q. This state is called the FFLO or LOFF state, and as a direct consequence of the non-zero center-of-mass momentum, the superconducting order parameter oscillates in space. Larkin and Ovchinnikov found that the order parameter has the spatial structure like $\cos (\mathbf{q} \cdot \mathbf{r})$ near the critical field in three dimensions rather than the structure like $\exp (\mathbf{i q} \cdot \mathbf{r})$ by calculations of the free energies of the states, although both structures have the same upper critical field within the second order transition.

The critical field of the FFLO state in three dimensions has a similar temperature dependence to the Pauli limit as mentioned above, wherease the value is slightly larger than the Pauli limit. Furthermore, the orbital pair-breaking effect strongly suppresses the FFLO state. The condition for the coexistence of the FFLO state and the vortex state was examined by Gruenberg and Gunther [22]. When the FFLO state coexists with the vortex state, the spatial oscillation of the order parameter due to the FFLO state is along the vortex lines.

On the other hand, in the two dimensions, the critical field as a function of the temperature is downwards convex $\left(\mathrm{d}^{2} H_{\mathrm{c} 2}(T) / \mathrm{d} T^{2}>0\right)$ at low temperatures 16, 8, ]7. This behavior, which is called upturn, is due to a Fermisurface effect analogous to nesting effects of spin and charge density waves [7, 9, 10,23. In the two dimensions, the Fermi-surfaces of up and down spin electrons touch on lines by a shift by $\mathbf{q}$ at $T=0$. It was found that by a calculation of the free energy that the order parameter has various two dimensional structures at low temperatures and high fields in the two dimensions [24]. The origin of the two dimensional structure is the high critical field, for which a gain in the polarization energy is more important than a loss in the condensation energy.

Temperature dependences similar to the theoretical prediction including the upturn have been observed in 
the organic superconductors [2,1, 3 6]. Hence, the FFLO state has often been discussed as a candidate to explain the critical field, although dimensional crossover effects in triplet and singlet superconductors are also possible mechanisms of the upturn 25 26]. However, the observation of the lower critical field in $\kappa$ (BEDT-TTF $)_{2} \mathrm{Cu}(\mathrm{NCS})_{2}$ 22], a change in the temperature dependence of upper critical field due to the purity of the samples of $\lambda$-(BETS $)_{2} \mathrm{GaCl}_{4}$ [4], and that due to tilt angle of the magnetic field in $\kappa$ (BEDT-TTF $)_{2} \mathrm{Cu}\left[\mathrm{N}(\mathrm{CN})_{2}\right] \mathrm{Br}$ [5 12,11] seem to support the FFLO state in these organic superconductors. In the phase diagram of $\lambda$-(BETS $)_{2} \mathrm{FeCl}_{4}$, the lower critical field of the field induced superconductivity shows upturnlike behavior $\left(\mathrm{d}^{2} H_{\mathrm{c} 2}(T) / \mathrm{d} T^{2}<0\right)$ which is naturally explained by the FFLO state in combination with the Jaccarino and Peter mechanism 27 29].

However, when we consider the FFLO state in the organic superconductors and the high- $T_{\mathrm{c}}$ cuprates, we should note that $d$-wave pairing is an important candidate of the pairing. The upturn of the critical field occurs also for the $d$-wave pairing as studied by Maki and Won [18] and many other authors [11, 19, 13]. However, in addition to the upturn at low temperatures $\left(T \lesssim 0.06 T_{\mathrm{c}}^{(0)}\right.$ ), the upper critical field $H_{\mathrm{c}}(T)$ exhibits a kink at $T \approx 0.06 T_{\mathrm{c}}^{(0)}$ due to a change in the direction of $\mathbf{q}$ [18,11, 19, 13]. When we assume $d_{x^{2}-y^{2} \text {-wave }}$ pairing, $\mathbf{q}$ is oriented to one of the crystal axes $(/ / x$ axis or $/ / y$-axis) for lower temperatures, whereas it is oriented to the direction of $(1,1,0)$ for higher temperatures $\left(0.06 T_{\mathrm{c}}^{(0)} \lesssim T \lesssim 0.56 T_{\mathrm{c}}^{(0)}\right)$. This behavior does not seem to be consistent with the experimental data of the upper critical fields [2], 3 6].

Here, we should add a discussion for the case in which the vortex state coexists with the FFLO state in the presence of the orbital pair-breaking effect. In this case, $\mathbf{q}$ of the FFLO state is fixed in the direction of the vortex line, and hence the kink does not occur. However, the temperature dependence of the critical field would change completely depending on the direction of the magnetic field within the directions parallel to the layers.

In this paper, we examine the case in which the orbital pair-breaking effect is negligible and $\mathbf{q}$ is freely oriented to the optimum direction at each temperature and field. The orbital pair-breaking effect is negligible, when separation of adjacent layers is sufficiently large [25], the layers are sufficiently thin [30], and the magnetic field is exactly aligned in a direction parallel to the layers [11,9]. It is shown that anisotropy of the Fermi-surface locks the optimum direction of $\mathbf{q}$, and thus the kink of $H_{\mathrm{c}}(T)$ disappears. We examine a tight-binding model on the square lattice 89.26] as an example of an anisotropic system. By change of the hole concentration, the shape of the Fermi-surface can be controlled between square and circular shapes.

At zero temperature, the hole concentration dependence of the critical field has been examined in the square lattice tight-binding model [9]. At hole concentration per a site $n_{\mathrm{h}} \approx 0.630$, (i.e., electron density per a site $n \approx 0.370$ ), the nesting is most effective, and the critical field is extraordinarily enhanced near this hole concentration. The Pauli paramagnetic limit $H_{\mathrm{P}}^{(0)}$ was also calculated in this paper, and it was found that the FFLO critical field exceeds $H_{\mathrm{P}}^{(0)}$ at all hole concentrations except in a small region near the half-filling.

In the present theory, we concentrate ourselves on the temperature dependence of the upper critical field. We consider sufficiently weak coupling of the pairing interaction and assume implicite small inter-layer electron hopping, which makes the mean field treatment appropriate.

The gap equation that we examine is

$$
\Delta_{\mathbf{q}}=\frac{V}{N} \sum_{\mathbf{k}}\left[\gamma_{\alpha}(\mathbf{k})\right]^{2} \frac{1-f\left(E_{\mathbf{k} \uparrow}\right)-f\left(E_{\mathbf{k} \downarrow}\right)}{2 E_{\mathbf{k}}} \Delta_{\mathbf{q}}
$$

where $f(\epsilon)$ is the Fermi distribution function, $\gamma_{\alpha}(\mathbf{k})$ is a symmetry factor of the gap function, $E_{\mathbf{k}} \equiv$ $\sqrt{\epsilon_{\mathbf{k} \sigma}^{2}+\left[\Delta_{\mathbf{g}} \gamma_{\alpha}(\mathbf{k})\right]^{2}}, \quad E_{\mathbf{k} \sigma} \equiv E_{\mathbf{k}}+\sigma h$, and $h \equiv$ $\mu_{0}|\mathbf{H}|[7,10,11,13]$. By an approximation of the standard weak coupling theory, we obtain

$$
\begin{aligned}
& \log \frac{T_{\mathrm{c}}^{(0)}}{T}=\int_{0}^{\infty} \mathrm{d} y \int_{-\pi}^{\pi} \frac{\mathrm{d} \theta}{2 \pi} \frac{\rho_{\alpha}(0, \theta)}{N_{\alpha}(0)} \\
& \times \sinh ^{2} \frac{\beta \zeta}{2} \frac{\tanh y}{y\left(\cosh ^{2} y+\sinh ^{2} \frac{\beta \zeta}{2}\right)},
\end{aligned}
$$

where

$$
\begin{aligned}
\zeta & \equiv h\left(\frac{\mathbf{v}_{\mathbf{F}} \cdot \mathbf{q}}{2 h}-1\right), \\
\rho_{\alpha}(\epsilon, \theta) & =\rho(\epsilon, \theta)\left[\left[\gamma_{\alpha}(\mathbf{k})\right]^{2}\right]_{|\mathbf{k}|=k_{\mathrm{F}}(\theta)} \\
N_{\alpha}(\epsilon) & =\int_{-\pi}^{\pi} \frac{\mathrm{d} \theta}{2 \pi} \rho_{\alpha}(\epsilon, \theta),
\end{aligned}
$$

and $\mathbf{v}_{\mathrm{F}}$ is the Fermi velocity. Here, $\rho(\epsilon, \theta)$ is the angle dependent density of states which satisfies

$$
\frac{1}{N} \sum_{\mathbf{k}} F\left(\epsilon_{\mathbf{k}}\right)=\int \mathrm{d} \epsilon \int_{-\pi}^{\pi} \frac{\mathrm{d} \theta}{2 \pi} \rho(\epsilon, \theta) F(\epsilon)
$$

for any function $F\left(\epsilon_{\mathbf{k}}\right)$, and $k_{\mathrm{F}}(\theta)$ is the magnitude of the Fermi momentum in the direction of $\theta$, which is the angle measured from the $k_{x}$-axis. By solving equation eq. (2) with q fixed, we obtain a transition temperature $T(h, \mathbf{q})$ or a critical field $h(T, \mathbf{q})$ for the value of $\mathbf{q}$. The final result of the transition tempreature is given by

$$
T_{\mathrm{c}}(h)=\max _{\mathbf{q}}(T(h, \mathbf{q}))
$$

As we mentioned above, we use the square lattice tightbinding model

$$
\epsilon_{\mathbf{k}}=-2 t\left(\cos k_{x}+\cos k_{y}\right)-\mu
$$


to express various anisotropic Fermi-surfaces. The cylindrically symmetric Fermi-surface is obtained in the limit of $\mu \rightarrow-4 t$, whereas a square Fermi-surface is obtained in the limit of $\mu \rightarrow 0$. Figure 1 shows the shape of the Fermi-surfaces of $\mu=-0.5 t,-1.8 t$ and $-3 t$. In this model, $\zeta$ is written as $\zeta=h\left(\bar{q} x\left(\theta, \varphi_{\mathbf{q}}\right)-1\right)$ with $\bar{q}=t q / h$ and $x\left(\theta, \varphi_{\mathbf{q}}\right)=\left[\cos \varphi_{\mathbf{q}} \sin \left(k_{x}\right)+\sin \varphi_{\mathbf{q}} \sin \left(k_{y}\right)\right]_{|\mathbf{k}|=k_{\mathrm{F}}(\theta)}$, where $\varphi_{\mathbf{q}}$ is the angle between $\mathbf{q}$ and $k_{x}$-axis. We examine $d_{x^{2}-y^{2}}$-wave pairing

$$
\gamma_{d_{x^{2}-y^{2}}}(\mathbf{k})=\left[\cos k_{x}-\cos k_{y}\right]_{|\mathbf{k}|=k_{\mathrm{F}}(\theta)},
$$

which is more favorable than $d_{x y}$-wave pairing, because of the peak in the angle dependent density of state $\rho(\epsilon, \theta)$ near the saddle points of the electron dispersion relation.

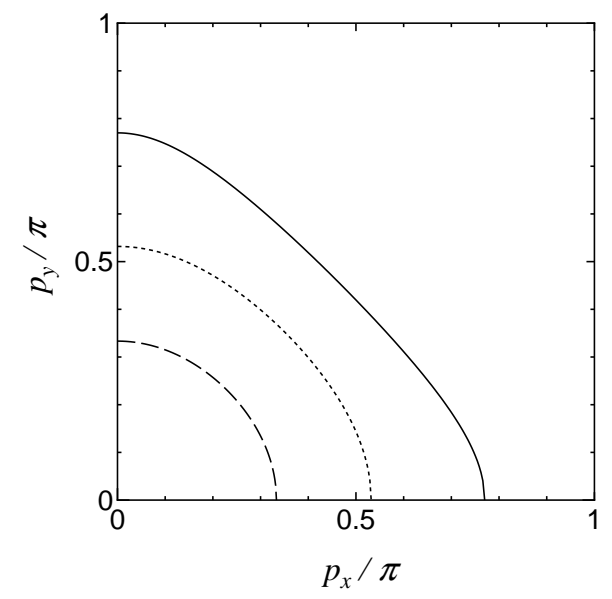

FIG. 1. The solid, dotted and dashed lines show the Fermi-surfaces at $\mu=-0.5 t,-1.8 t$ and $3 t$, respectively. The lattice constant is taken as unity.

Figure 2 shows the critical field for $\mu=-3 t$, i.e., $n \approx 0.17$. In the vertical axis, $h=\mu_{0}|\mathbf{H}|$ is scaled by $\Delta_{d 0} \equiv 2 \omega_{\mathrm{D}} \exp \left(-1 / V N_{d}(0)\right)$, which is rather different from the zero field BCS gap for anisotropic pairing [9]. As shown in Fig. 1, the Fermi-surface has a nearly cylindrical shape at this value of $\mu$. The behavior of the critical field is similar to that for the cylindrically symmetric Fermi-surface, which corresponds to $\mu \rightarrow-4 t$. The optimum direction of $\mathbf{q}$ of the FFLO state changes at a temperature $T \approx 0.1 T_{\mathrm{c}}^{(0)}$, where the kink appears. Weak anisotropy does not change this behavior. It is confirmed by numerical calculations that the other directions $\mathbf{q}$ than $\varphi_{\mathbf{q}}=0$ and $\pi / 4$ give lower critical fields than the highest one.

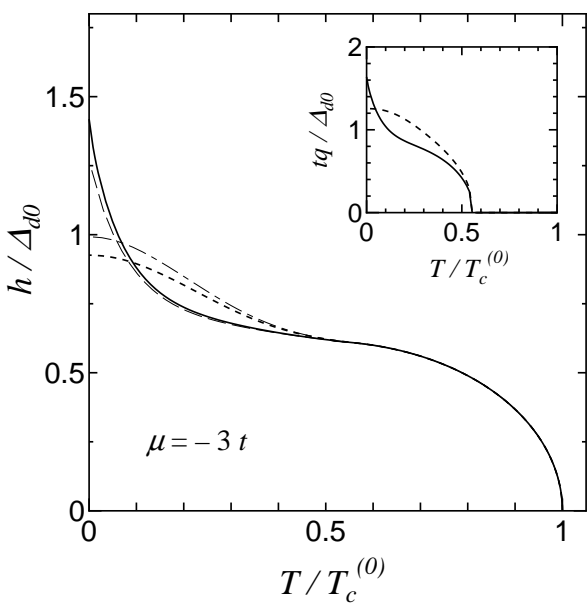

FIG. 2. The solid and dotted lines show the critical fields for $\varphi_{\mathbf{q}}=0$ and $\pi / 4$, respectively, when $\mu=-3 t$, i.e., $n \approx 0.17$. In the inset, the solid and dotted lines show the temperature dependences of $q=|\mathbf{q}|$ along the transition lines, for $\varphi_{\mathbf{q}}=0$ and $\pi / 4$, respectively. The dashed and dot-dashed lines show the results of cylindrical symmetric Fermi-surface, (i.e., $\mu \rightarrow-4 t$ ), for $\varphi_{\mathbf{q}}=0$ and $\pi / 4$, respectively.

Figure 3 shows the results for $\mu=-1.8 t$, i.e., $n \approx 0.41$. As shown in our previous paper [9], the critical field increases markedly near $\mu=-2 t$ due to an effect of the structure of the Fermi-surface in the present model. Therefore, for $\mu=-1.8 t$, the critical field is very large at $T=0$. It is found that the enhancement occurs especially at low temperatures, where $\varphi_{\mathbf{q}}=0$ is the optimum direction. At rather higher temperatures, $\varphi_{\mathbf{q}}=\pi / 4$ is the optimum direction, which gives critical fields slightly higher than that of $\varphi_{\mathbf{q}}=0$. As shown in Fig. 14, other directions are optimum near the kink, where the curves of $\varphi_{\mathbf{q}}=0$ and $\pi / 4$ cross, although the differences in the magnitudes of the critical fields are very small there. Such behavior does not occur for $\mu=-3 t$ and $\mu \rightarrow-4 t$.

Figure 5 shows the critical field for $\mu=-0.5 t$, i.e., $n \approx 0.77$, where the Fermi-surface has a nearly square shape. It is found that the optimum $\mathbf{q}$ is oriented to the direction of the crystal axis $\left(\varphi_{\mathbf{q}}=0\right)$ in a whole temperature region, and thus the kink does not occur. 


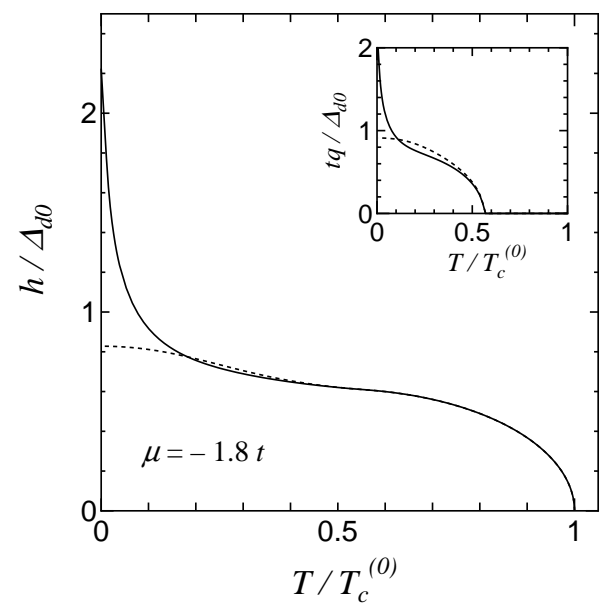

FIG. 3. The solid and dotted lines show the critical fields for $\varphi_{\mathbf{q}}=0$ and $\pi / 4$, respectively, when $\mu=-1.8 t$, i.e., $n \approx 0.41$. In the inset, the solid and dotted lines show the temperature dependences of $q=|\mathbf{q}|$ along the transition line for $\varphi_{\mathbf{q}}=0$ and $\pi / 4$, respectively.

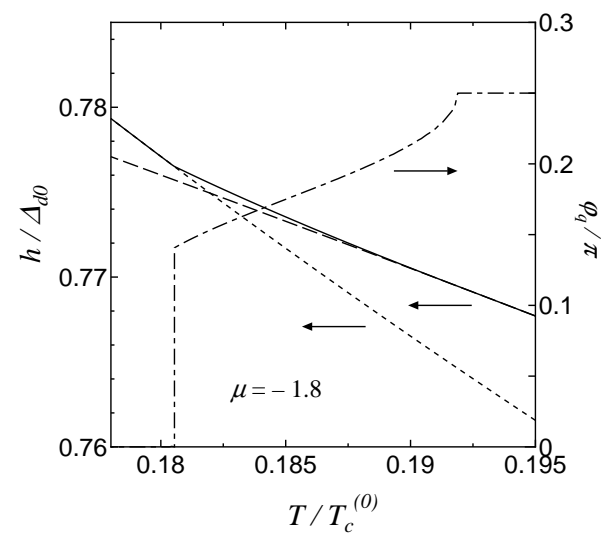

FIG. 4. Behaviors of the critical fields and $q=|\mathbf{q}|$ near the kink, where the lines of $\varphi_{\mathbf{q}}=0$ and $\pi / 4$ cross, when $\mu=-1.8 t$. The dotted and dashed lines show the critical fields of $\varphi_{\mathbf{q}}=0$ and $\pi / 4$, respectively. The solid line shows the final result of the critical field obtained by optimizing both the direction and the magnitude of $\mathbf{q}$. The dot-dashed line shows the optimum $\varphi_{\mathbf{q}}$.

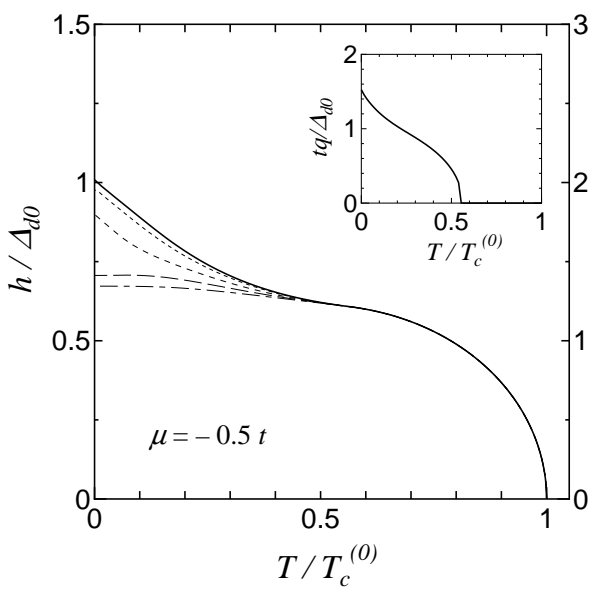

FIG. 5. The solid, dotted, short dashed, dashed and dot-dashed lines show the critical fields for $\varphi_{\mathbf{q}}=0, \pi / 20$, $\pi / 10,3 \pi / 20$, and $\pi / 4$, respectively, when $\mu=-0.5 t$, i.e., $n \approx 0.77$. The inset shows the temperature dependence of $q=|\mathbf{q}|$ along the transition line.

In conclusion, we have studied the upper critical field of the superconductor on the square lattice in parallel magnetic fields. In particular, we have examined anisotropy effects of Fermi-surface on the temperature dependence of the upper critical field. By changing the hole concentration, we have controlled the shape of the Fermisurface. The results are summarized as follows: (1) The kink of the critical field as a function of the temperature disappears due to an anisotropy of the Fermi-surface of some extent (Fig. 5); (2) When the anisotropy is weak, the kink remains (Fig. 2); (3) Depending on the shape of the Fermi-surface and the temperature, the optimum $\mathbf{q}$ is oriented to other directions than the symmetric directions $\varphi_{\mathbf{q}}=0$ and $\pi / 4$ (Fig. A); (4) The critical field increases rapidly especially at low temperatures (Fig. 3) near $\mu \approx-2 t$, where the nesting is most effective [9. From the result (1), the absence of the kink in the experimental data which exhibits the upturn at low temperatures does not exclude the possibility of the FFLO state of anisotropic singlet pairing.

When the orbital pair-breaking effect remains to some extent, for example, for large inter-layer coupling, the present result must be taken in another way. In this case, the direction of $\mathbf{q}$ coincides with the direction of the vortex lines for the coexistence of the vortex state and the FFLO state. Hence, $\varphi_{\mathbf{q}}$ must be regarded as the direction of the applied magnetic field in Figures 2, 3 and 5. The temperature dependence of the upper critical field changes qualitatively as shown in those figures, by change of the direction of the applied field between $(1,0,0)$ and $(1,1,0)$ directions, when the vortex state coexists. 
[1] T. Ishiguro: J. Supercond. 13 (2000) 817; J. Phys. IV, Proc. (France), 10 (2000) 139, and references therein.

[2] J. Singleton, J. A. Symington, M.-S. Nam, A. Ardavan, M. Kurmco, and P. Day: J. Phys. 12 (2000) L641; J. A. Symington, J. Singleton, M.-S. Nam, A. Ardavan, M. Kurmco, and P. Day: Physica B 294-295 (2001) 418.

[3] E. Ohmichi, Y. Shimojo, T. Ishiguro, T. Sakon, T. Sasaki, M. Motokawa, R. B. Lyubovskii and R. N. Lyubovskaya: Synth. Met. 103 (1999) 2107; Y. Shimojo, A. E. Kovalev, S. Kamiya, E. Ohmichi, T. Ishiguro, H. Yamochi, G. Saito, A. Ayari, and P. Monceau: Physica B 294-295 (2001) 427.

[4] M. A. Tanatar, T. Ishiguro, H. Tanaka, H. Kobayashi: unpublished.

[5] E. Ohmichi: unpublished.

[6] I. J. Lee, M. J. Naughton, G. M. Danner and P. M. Chaikin: Phys. Rev. Lett. 78 (1997) 3555.

[7] H. Shimahara: Phys. Rev. B 50 (1994) 12760.

[8] H. Burkhardt and D. Rainer: Ann. Physik 3 (1994) 181.

[9] H. Shimahara: J. Phys. Soc. Jpn. 68 (1999) 3069; Journal of Superconductivity, 12 (1999) 469-473.

[10] H. Shimahara: J. Phys. Soc. Jpn. 66 (1997) 541.

[11] H. Shimahara and D. Rainer: J. Phys. Soc. Jpn. 66 (1997) 3591; U. Klein, D. Rainer, and H. Shimahara: J. Low Temp. Phys. 118 (2000) 91.

[12] S. Manalo and U. Klein: J. Phys. 12 (2000) L471.

[13] H. Shimahara: Phys. Rev. B 62 (2000) 3524.

[14] P. Fulde and R. A. Ferrell: Phys. Rev. 135 (1964) A550.

[15] A. I. Larkin and Yu. N. Ovchinnikov: Zh. Eksp. Teor. Fiz. 47 (1964) 1136; translation: Sov. Phys. JETP, 20 (1965) 762 .

[16] L. N. Bulaevskii: Zh. Eksp. Teor. Fiz. 65 (1973) 1278, translation: Sov. Phys. JETP 38 (1974) 634.

[17] K. Aoi, W. Dieterich and P. Fulde: Z. Phy. 267 (1974) 223.

[18] K. Maki and H. Won: Czechoslovak J.Phys. 46 (1996) Suppl. S2, 1035.

[19] K. Yang and S. L. Sondhi: Phys. Rev. B 57 (1998) 8566.

[20] B. S. Chandrasekhar: App. Phys. Lett. 1 (1962) 7.

[21] A. M. Clogston: Phys. Rev. Lett. 9 (1962) 266.

[22] L. W. Gruenberg and L. Gunther: Phys. Rev. Lett. 16 (1966) 996.

[23] H. Shimahara and S. Hata: Phys. Rev. B 62 (2000) 14541.

[24] H. Shimahara: J. Phys. Soc. Jpn. 67 (1998) 736.

[25] A. G. Lebed: Phys. Rev. B 59 (1999) R721; A. G. Lebed: Pis'ma Zh. Eksp. Teor. Phys. 44 (1986) 89 [JETP Lett. 44 (1986) 114]; A. G. Lebed and K. Yamaji: Phys. Rev. Lett. 80 (1998) 2697.

[26] M. Miyazaki, K. Kishigi and Y. Hasegawa: J. Phys. Soc. Jpn. 68 (1999) 2344.

[27] S. Uji, H. Shinagawa, T. Terashima, T. Yakabe, Y. Terai, M. Tokumoto, A. Kobayashi, H. Tanaka and H. Kobayashi: Nature 410 (2001) 908; unpublished.

[28] V. Jaccarino and M. Peter: Phys. Rev. Lett. 9 (1962) 290.

[29] H. Shimahara: to be published in J. Phys. Soc. Jpn. 71, No.3 (2002).

[30] S. Manalo and U. Klein: unpublished. 